\title{
Prolonged mechanical unloading preserves myocardial contractility but impairs relaxation in rat heart of dilated cardiomyopathy accompanied by myocardial stiffness and apoptosis
}

\author{
Hiroyuki Muranaka, MD, Akira Marui, MD, PhD, Masaki Tsukashita, MD, PhD, Jian Wang, MD, PhD, \\ Jota Nakano, MD, Tadashi Ikeda, MD, PhD, and Ryuzo Sakata, MD, PhD
}

\begin{abstract}
Objective: Left ventricular assist devices are used in patients with end-stage dilated cardiomyopathy as a "bridge to recovery." However, physiologic and histologic changes under prolonged mechanical unloading have not been elucidated. Thus, we investigated these changes in the rat heart with dilated cardiomyopathy under mechanical unloading after heterotopic transplantation.
\end{abstract}

\begin{abstract}
Methods: Six weeks after induction of autoimmunized dilated cardiomyopathy in Lewis rats, 2 types of hearts were compared ( $\mathrm{n}=6$ each): (1) an unloaded dilated cardiomyopathy heart (DCM-UL) and (2) a dilated cardiomyopathy heart (DCM). The hearts were evaluated 2 and 4 weeks after transplantation.
\end{abstract}

\begin{abstract}
Results: Four weeks after transplantation, developed tension of the papillary muscle (indicator of myocardial contractility) and $\beta$-adrenergic response to isoproterenol were better in DCM-UL than in DCM $(P=0.0025$ and $P<0.0001$, respectively). However, half-relaxation time of the papillary muscle (indicator of myocardial relaxation) was worse in the DCM-UL group $(P<.0001)$. The ratio of the fibrotic area of the myocardium and the number of terminal dUTP nick end-labeling-positive myocytes (indicator of myocardial apoptosis) were higher in DCM-UL than in DCM ( $P=.0072$ and $P=.0039$, respectively). The mRNA expression of collagen Ia was also higher in DCM-UL.
\end{abstract}

Conclusions: Mechanical unloading preserved myocardial contractility and $\beta$-adrenergic response but worsened myocardial relaxation. Furthermore, prolonged mechanical unloading has a tendency to increase the ratio of the fibrotic area and myocardial apoptosis. These unfavorable responses, although secondary to prolonged mechanical unloading, may have a negative impact on the bridge to recovery in patients with dilated cardiomyopathy. (J Thorac Cardiovasc Surg 2010;140:916-22)

Use of a left ventricular assist device (LVAD) has become an established procedure for patients with end-stage heart failure. In some cases, the LVAD enables recovery from a decompensating heart. ${ }^{1}$ Several investigations have demonstrated a recurrence of heart failure after LVAD explantation. ${ }^{2}$ Prolonged unloading also induces depression of ventricular contractility owing to complicated histologic and physiologic changes in the myocardium. ${ }^{3}$ In an experimental study, we demonstrated that mechanical unloading of the left ventricle (LV) can improve ventricular contractility and $\beta_{1^{-}}$and $\beta_{2}$-adrenergic receptor (AR) gene expression. However, the recovery did not last long in the rat model with ischemic cardiomyopathy. ${ }^{4-6}$

From the Department of Cardiovascular Surgery, Kyoto University Graduate School of Medicine, Kyoto, Japan.

Disclosures: None.

Received for publication Sept 3, 2009; revisions received Jan 8, 2010; accepted for publication Feb 2, 2010; available ahead of print April 12, 2010.

Address for reprints: Akira Marui, MD, PhD, Department of Cardiovascular Surgery,

Kyoto University Graduate School of Medicine, 54 Shogoin-Kawahara, Sakyo,

Kyoto 606-8507, Japan (E-mail: marui@kuhp.kyoto-u.ac.jp).

$0022-5223 / \$ 36.00$

Copyright (c) 2010 by The American Association for Thoracic Surgery

doi: $10.1016 /$ j.jtcvs. 2010.02 .006
Dilated cardiomyopathy (DCM) presents end-stage heart failure with severe systolic dysfunction and diffuse interstitial fibrosis. However, the physiologic and histologic changes of DCM under mechanical unloading have not been well demonstrated. To date, no study has systematically examined LVADs with DCM as a "bridge to recovery." Therefore, the present study aimed to investigate the changes under mechanical unloading in the rat model with DCM using heterotopic transplantation.

\section{MATERIALS AND METHODS Rat DCM Model}

All experimental procedures were conducted according to the guidelines for animal care stated by Kyoto University and the "Guide for the Care and Use of Laboratory Animals,"' published by the National Institutes of Health.

Five-week-old male Lewis rats (weighing 120-150 g; Japan SLC Inc, Hamamatsu, Japan) were used. DCM was produced by inducing experimental myocarditis. ${ }^{7}$ In brief, $1 \mathrm{mg} / 0.1 \mathrm{~mL}$ of purified cardiac myosin from a porcine heart was mixed with an equal volume of Freund's complete adjuvant (Difco; BD Diagnostic Systems, Sparks, Md) and injected into a footpad. Six weeks after immunization, these rats served as a model of heart failure owing to DCM.

\section{Echocardiography}

The rats were anesthetized with ether 6 weeks after autoimmunization. LV function was evaluated by echocardiography with a $12-\mathrm{MHz}$ phased 


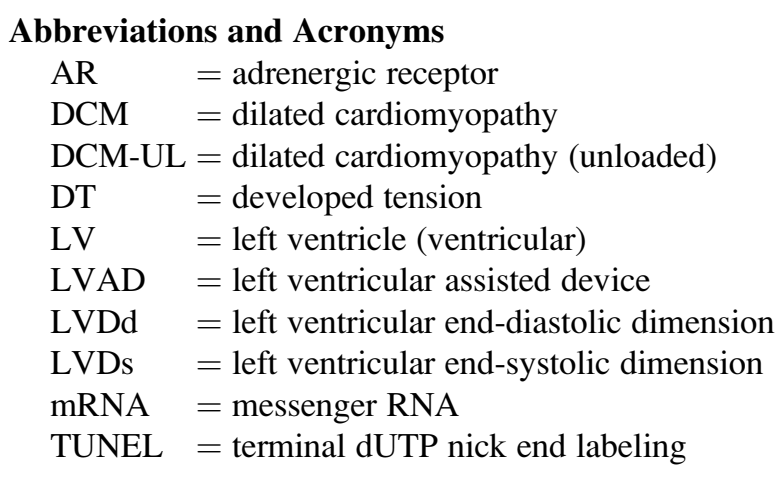

array transducer (HP SONOS 4500; Agilent Technologies, Andover, Mass). The following parameters were measured by M-mode tracing: LV end-diastolic dimension (LVDd) and LV end-systolic dimension (LVDs). Fractional shortening was calculated by the following equation: (LVDd LVDs)/LVDd $\times 100$

\section{Heterotopic Transplantation}

The heart and lungs of DCM rats were heterotropically transplanted into recipient rats to establish mechanical unloading, as described previously. ${ }^{6,8}$ In brief, the DCM heart and lungs were harvested after ligation of the left and right superior venae cavae and inferior venae cavae. The ascending aorta of the donor heart was anastomosed end to side to the abdominal aorta of the recipient. After heterotopic transplantation, the rats survived with an operative mortality of less than $5 \%$.

\section{Study Groups}

After echocardiographic examination, the rats in which DCM developed with an LV fractional shortening within $15 \%$ to $40 \%$ were then divided into the following 2 groups: (1) the DCM-unloading (DCM-UL) group, which had the DCM heart and lungs heterotopically transplanted into the recipient rats $(n=6)$, and (2) the DCM group, which had the DCM heart without transplantation $(n=6)$. The hearts were excised and evaluated at 2 and 4 weeks after transplantation. The hearts of normal Lewis rats served as the control group $(n=6)$.

\section{Papillary Muscle Tests}

Contraction and relaxation. Papillary muscle function was examined at experiment end points as described previously. ${ }^{5,6,8,9}$ After the rats were anesthetized and heparinized, the transplanted hearts in the DCM-UL group and the DCM or normal hearts in the nontransplanted group were rapidly removed. Each heart was placed into normal Tyrode solution. Posterior papillary muscle was carefully ligated with a silk thread, dissected from the LV wall, and mounted in a tissue bath containing Krebs-Henseleit solution. The bath was maintained at a temperature of $37^{\circ} \mathrm{C}$ and bubbled with oxygen (95\%) and carbon dioxide (5\%). The papillary muscle was stimulated at $1 \mathrm{~Hz}$ with impulses of $5 \mathrm{~ms}$ in duration and 5 -ms pulses at a voltage approximately $10 \%$ above the threshold level. The papillary muscle was stretched to the length at which maximum tension occurred. After stabilization, isometric tension and half-relaxation time were recorded digitally at the maximum tension position. Developed tension (DT) was normalized to the cross-sectional area. DT and half-relaxation time indicate myocardial contractility and relaxation, respectively.

Responses to isoproterenol. After baseline measurements were completed, DT was recorded during exposure to the $\beta$-adrenergic agonist isoproterenol. Isoproterenol $\mathrm{HCl}$ was dissolved in distilled water and added to the tissue bath to produce a cumulative concentration of $10^{-8}, 10^{-7}$, and $10^{-6} \mathrm{~mol} / \mathrm{L}$. DT was measured when the response was maximal (5-10 minutes after each addition of isoproterenol).

\section{Histology}

The papillary muscle was removed from the heart; the LV myocardium was transversely sliced into sections $(2 \mathrm{~mm}$ in diameter) at the base of the papillary muscle and then fixed in $10 \%$ buffered formalin. The remaining LV myocardium was frozen at $-80^{\circ} \mathrm{C}$ until analyzed. Transverse sections of the LV myocardium were stained with hematoxylin-eosin and picrosirius red reagents. Mean cardiomyocyte diameters were calculated by measuring 20 cells in the LV myocardium under microscope (magnification $\times 200$ ) as described previously. ${ }^{5,6,8,9}$ By means of picrosirius red staining, the percentage of the fibrotic area was calculated with an IPLab for the Windows image system (Solution Systems, Inc, Chiba, Japan).

The transverse sections were also used for in situ detection of apoptosis by the terminal dUTP nick end labeling (TUNEL) assay. TUNEL assay was performed according to the manufacturer's protocol (Takara Biomedicals Inc, Shiga, Japan) as described previously. ${ }^{9}$ TUNEL-positive cells were counted in a blinded fashion, with a maximum of 10 randomly chosen fields in each section.

\section{Analysis of Messenger RNA (mRNA) Expression}

Total messenger RNA (mRNA) was prepared from the frozen LV pieces with TRIzol reagent (Life Technologies Corporation, Carlsbad, Calif), reverse transcribed, and amplified with the ABI PRISM 7300 sequence detector (Applied Biosystems, Inc, Foster City, Calif). Polymerase chain reaction conditions included 40 cycles of denaturing at $94^{\circ} \mathrm{C}$ for 20 seconds and primer annealing/extension at $62^{\circ} \mathrm{C}$ for 60 seconds. The polymerase chain reaction sequence of $\beta_{1}$ - and $\beta_{2}$-AR, collagen Ia, and caspase-3 were reported in our previous research. ${ }^{5,6,8,9}$ The TaqMan rodent glyceraldehyde3-phosphate dehydrogenase control reagent was used to detect rat glyceraldehyde-3-phosphate dehydrogenase as the internal standard. The expression level of the target gene was normalized to glyceraldehyde-3phosphate dehydrogenase level in each sample.

\section{Statistical Analysis}

All data are described as mean \pm SEM. Differences among groups were evaluated by the Student $t$ test. Two-way repeated measures analysis of variance was used to assess the inotropic response of the papillary muscle to isoproterenol. All statistical analyses were performed with Statview for Windows version 5.0 (SAS Institute, Inc, Cary, NC). Statistical significance was accepted at the level of $P<.05$.

\section{RESULTS}

\section{Development of DCM}

DCM developed in 30 of the 80 rats 6 weeks after autoimmunization (Table 1). LVDd and LVDs in the DCM group were larger than those in the control group. LV posterior wall depth and fractional shortening in the DCM group were smaller than those in the controls.

\section{Weight and Cardiomyocyte Diameter}

LV weight in the DCM-UL group after unloading was lower than in the DCM group at 2 and 4 weeks $(P<.0001$, respectively), as shown in Table $2 . \mathrm{LV}$ myocardial diameter in the DCM-UL group after unloading was also smaller than that in the DCM at 2 and 4 weeks $(P=.0001, P<.0001$, respectively $)$, as shown in Table 2 . These results indicated that mechanical unloading after 
TABLE 1. Baseline echocardiographic data

\begin{tabular}{lcc}
\hline & $\begin{array}{c}\text { Control group } \\
(\mathbf{n}=\mathbf{6})\end{array}$ & $\begin{array}{c}\text { DCM group } \\
(\mathbf{n}=\mathbf{2 4})\end{array}$ \\
\hline LVDd $(\mathrm{mm})$ & $7.3 \pm 0.2$ & $8.4 \pm 0.2^{*}$ \\
LVDs $(\mathrm{mm})$ & $3.2 \pm 0.2$ & $6.1 \pm 0.2^{*}$ \\
PWd $(\mathrm{mm})$ & $1.5 \pm 0.2$ & $1.1 \pm 0.0^{*}$ \\
FS $(\%)$ & $56.8 \pm 1.8$ & $27.7 \pm 1.4^{*}$ \\
\hline LVDd, Left ventricular end-diastolic dimension; $L V D s$, left ventricular end-systolic \\
dimension; $P W d$, posterior wall depth; $F S$, fractional shortening; DCM, dilated cardio- \\
myopathy. Data are presented as mean \pm SEM. *Compared with the control group \\
$(P<.05)$.
\end{tabular}

heterotopic transplantation induced myocardial atrophy and that the atrophy progressed with time.

\section{Papillary Muscle Tests}

DT and half-relaxation time are shown in Figure 1, $A$ and $B$, respectively. The DT of papillary muscle after transplantation was higher in the DCM-UL group than in the DCM group at 2 and 4 weeks $(P=.0016, P=.0025$, respectively). Half-relaxation time of the papillary muscle was higher in the DCM-UL group than in the DCM group at 2 and 4 weeks $(P=.017, P<.0001$, respectively $)$.

$\beta$-adrenergic response of the papillary muscle is presented in Figure 1, C. Inotropic responses to isoproterenol $\left(10^{-8}\right.$, $10^{-7}$, and $10^{-6} \mathrm{~mol} / \mathrm{L}$ ) after unloading were higher in the DCM-UL group than in the DCM group at 2 and 4 weeks $(P<.0001)$.

\section{Histology}

Myocardial fibrotic area. Myocardial fibrotic area stained with picrosirius red is shown in Figure 2. There was no difference in the ratio of the fibrotic area between the DCM-UL group and the DCM group at 2 weeks after unloading. However, the ratio of the fibrotic area in the DCM-UL group was larger than in the DCM group 4 weeks after unloading $(P=.0072)$.

TUNEL-positive cardiomyocyte. The TUNEL-positive myocyte count (indicator of cardiac apoptosis) is shown in Figure 2. There was no difference in TUNEL-positive myocytes between the DCM-UL and DCM groups $(P=.099) 2$ weeks after unloading. However, 4 weeks after unloading, the number of TUNEL-positive myocytes in the DCM-UL group was higher than that in the DCM group $(P=.0039)$. These results indicated that myocardial apoptosis progressed with time under mechanical unloading in the rat DCM heart.

\section{Analysis of mRNA Expression}

The mRNA expression of $\beta_{1}$-ARs, $\beta_{2}$-ARs, and collagen Ia was higher in the DCM-UL group than in the DCM group 2 weeks after transplantation (Table 3 ). There was no difference in caspase- 3 mRNA expression between the 2 groups.

\section{DISCUSSION \\ Main Findings}

To our knowledge, this is the first study to investigate the physiologic and histologic changes in mechanical unloading of the rat DCM heart. The present study demonstrated the following novel findings in the rat DCM model with heterotopic transplantation: mechanical unloading preserved myocardial contractility and response to $\beta$-adrenergic stimulation; however, unloading impaired myocardial relaxation. Mechanical unloading also increased myocardial atrophy and the ratio of the fibrotic area and apoptosis with time. Evaluation with mRNA expression was consistent with these results. Thus, prolonged mechanical unloading may preserve myocardial contractility and response to $\beta$-adrenergic stimulation, but it impairs myocardial relaxation and facilitates myocardial stiffness and apoptosis.

\section{Unloading and Myocardial Contractility}

Many investigations have revealed that mechanical unloading with LVAD induces the myocardial recovery of systolic functions in patients with end-stage heart failure. ${ }^{1,10,11}$ LVAD support shifts leftward the slope of the pressure-volume loop and increases maximum energy owing to volume and pressure unload of the LV. ${ }^{12}$ In clinical and animal studies using isolated myocardium, trabeculae, and papillary muscle, mechanical unloading with LVAD support and heterotopic transplantation has improved fractional shortening, time to peak contraction, and the force-frequency relationship. ${ }^{10,12,13}$ LVAD support has also normalized $\beta$-adrenergic response and $\beta$-AR density. Furthermore, it has also upregulated gene expression for

TABLE 2. Morphologic and histologic studies

\begin{tabular}{|c|c|c|c|c|c|}
\hline & \multirow[b]{2}{*}{ Control } & \multicolumn{2}{|c|}{ Two weeks } & \multicolumn{2}{|c|}{ Four weeks } \\
\hline & & DCM & DCM-UL & DCM & DCM-UL \\
\hline LV weight (g) & $0.71 \pm 0.29$ & $0.96 \pm 0.51 *$ & $0.57 \pm 0.12 \dagger$ & $0.96 \pm 0.33 *$ & $0.47 \pm 0.37^{*}, \dagger$ \\
\hline Myocytes diameter $(\mu \mathrm{m})$ & $21.3 \pm 1.2$ & $30.0 \pm 1.3^{*}$ & $19.9 \pm 1.0 \dagger$ & $32.0 \pm 0.6^{*}$ & $16.6 \pm 0.5^{*}, \dagger$ \\
\hline Fibrotic area $(\%)$ & $0.5 \pm 0.1$ & $16.2 \pm 2.5^{*}$ & $19.3 \pm 3.5^{*}$ & $12.0 \pm 1.9^{*}$ & $22.3 \pm 2.4^{*}, \dagger$ \\
\hline $\begin{array}{l}\text { TUNEL-positive } \\
\text { cardiomyocyte }\left(/ 10^{4}\right)\end{array}$ & $0.35 \pm 0.14$ & $3.43 \pm 0.52 *$ & $4.77 \pm 0.52 *$ & $2.13 \pm 0.23 *$ & $3.35 \pm 0.23 *, \dagger$ \\
\hline
\end{tabular}

$L V$, Left ventricle; $T U N E L$, terminal dUTP nick end-labeling; $D C M$, dilated cardiomyopathy; $D C M$ - $U L$, dilated cardiomyopathy (unloaded) $* P<.05$ vs control group. $\dagger P<.05$ vs DCM at the same time point. 

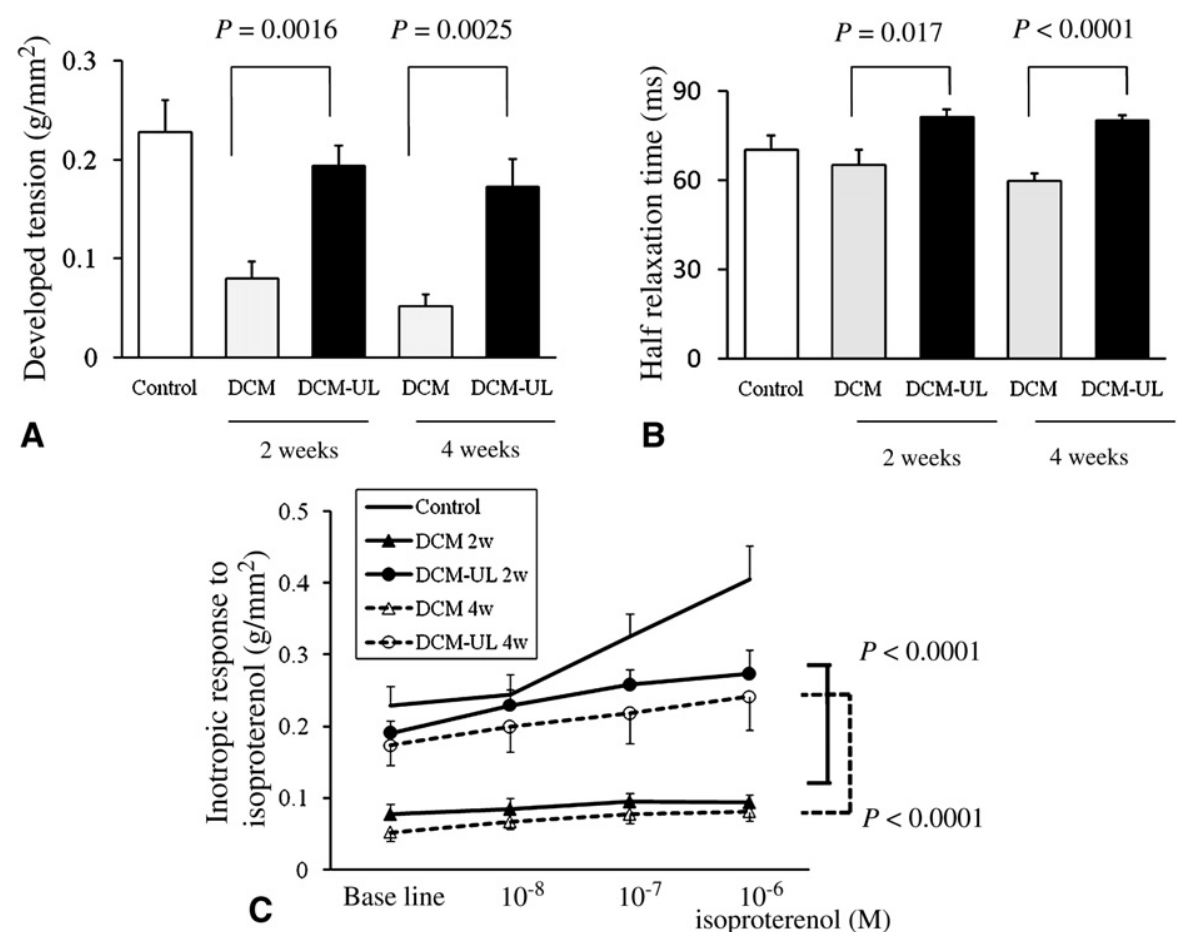

FIGURE 1. Analysis of changes in DT, half-relaxation time, and inotropic response to isoproterenol with isolated papillary muscle at 2 and 4 weeks after transplantation. All values are represented as mean \pm SEM. $D T$, Developed tension; $D C M$, dilated cardiomyopathy; $D C M$ - $U L$, dilated cardiomyopathy (unloaded).

the sarcoplasmic endoreticular $\mathrm{Ca}^{2+}$-adenosine triphosphatase subtype 2a, the ryanodine receptor, and the sarcolemmel $\mathrm{Na}^{+}-\mathrm{Ca}^{2+}$ exchanger. ${ }^{14}$

Despite the recovery of systolic function, prolonged mechanical unloading with LVAD support has not always yielded positive results. Xydas and associates ${ }^{3}$ reported that cardiac recovery peaked at 60 days.

In the present study, mechanical unloading preserved the DT of the papillary muscle and the inotropic response to $\beta$-adrenergic stimulation in rats with DCM for 4 weeks after transplantation. These results are consistent with our previous reports in the rat model with ischemic cardiomyopaathy., Preservation of myocardial contractility may relate to some other mechanisms. In our study, an analysis of the $\beta_{1^{-}}$and $\beta_{2}$-AR mRNA expression demonstrated that mechanical unloading upregulated the $\beta$-AR gene expression, and it was compatible with the papillary muscle test.

These findings suggest that mechanical unloading can have a substantial impact on hemodynamics and cardiac contractility by upregulation of $\beta$-ARs. This mechanism is one of the most important factors in the case for a bridge to recovery for patients with end-stage heart failure. Thus, further studies are needed to clarify how long the contractile recovery could last after mechanical unloading.

\section{Unloading and Myocardial Relaxation}

Early investigation has revealed that LVAD support shifts the end-diastolic pressure-volume relationship slope left- ward and increases myocardial stiffness. ${ }^{12}$ In a similar report, Brinks and associates ${ }^{15}$ described an animal model with 30 days of mechanical unloading.

We evaluated the half-relaxation time of the isolated papillary muscle as an indicator of myocardial relaxation. Welsh and colleagues ${ }^{13}$ reported that the half-relaxation time of an isolated cardiomyocyte was unchanged after 2 weeks of mechanical unloading of the nonfailing heart in the rat model. On the other hand, Soppa and coworkers ${ }^{16}$ demonstrated the prolongation of half-relaxation time after 5 weeks of mechanical unloading in the normal rat heart.

In the present study, half-relaxation time in the DCM-UL group was prolonged compared with that of the DCM group 2 and 4 weeks after transplantation. Histologic assessment revealed that the ratio of the fibrotic area of the myocardium was more prominent in the DCM-UL group than in the DCM group from the early phase onward.

\section{Myocardial Fibrotic Area}

Whether myocardial fibrosis progresses under mechanical unloading has been a source of controversy. Bruckner and coworkers ${ }^{17}$ reported that prolonged mechanical unloading reduces myocardial fibrosis. On the other hand, many reports have shown that mechanical unloading increases interstitial fibrosis. ${ }^{4-6,18}$ Klotz and associates ${ }^{19}$ demonstrated an increase in LV cross-linked collagen content and tissue angiotensin I and II levels after LVAD support in DCM. In 

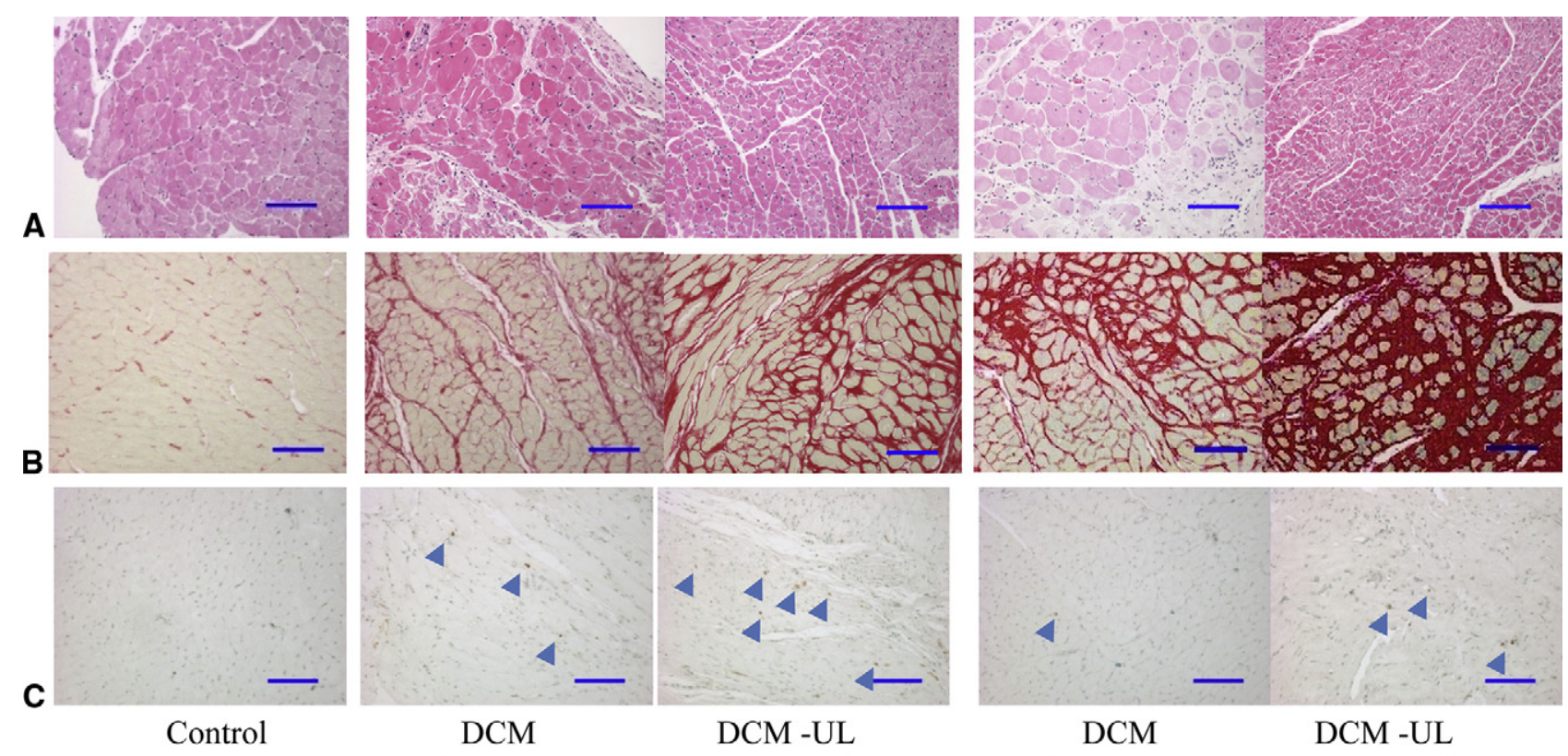

2 weeks

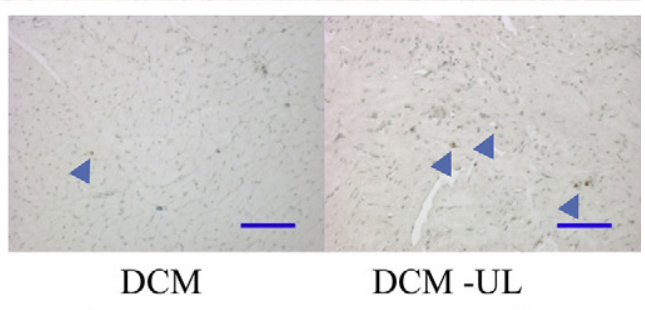

4 weeks

FIGURE 2. A, Hematoxylin-eosin stained sections of the LV myocardium at 2 and 4 weeks after transplantation. B, Picrosirius red-stained sections of the LV myocardium. C, TUNEL-stained sections of the LV myocardium. Arrow indicates TUNEL-positive cardiomyocyte (bar $=100 \mu \mathrm{m}) . L V$, Left ventricle; $T U N E L$, terminal dUTP nick end labeling; $D C M$, dilated cardiomyopathy; $D C M-U L$, dilated cardiomyopathy (unloaded).

relation to fibrosis and the duration of mechanical unloading, Bruggink and colleagues ${ }^{20}$ reported that extracellular matrix volume increased during the first 200 days after LVAD implantation; after 200 days, extracellular matrix volume decreased with time.

In the present study, picrosirius red staining highlighted the ratio of the fibrotic area in unloading hearts. We believe that this finding is a result of certain histologic mechanisms. Fibrotic tissue does not reduce in parallel with cardiomyocyte volume. Therefore, an uneven change in myocardial content may contribute to heighten the ratio of the fibrotic area after unloading. Similar findings have been presented in prior studies. ${ }^{19,21}$ We believe that an increase in the ratio of fibrotic tissue plays an important role in the stiffness of isolated papillary muscle. Moreover, this may exert a negative influence on LV systolic and diastolic function.

\section{Myocardial Apoptosis}

Schena and coworkers ${ }^{22}$ demonstrated an increase in caspase-3 activity after mechanical unloading in the rat model with a normal heart. $\mathrm{We}^{9}$ previously reported that mechanical unloading increased TUNEL-positive myocytes and upregulated mRNA expression of caspase- 3 in the rat ischemic cardiomyopathy model. In the present study, caspase-3 gene expression was higher in the DCM-UL group than in the DCM group, and the TUNEL-positive myocyte cell count was higher than that in the DCM group at 4 weeks. These results indicate that mechanical unloading may induce myocardial apoptosis. On the other hand, several studies have demonstrated an antiapoptotic effect after LVAD implantation. ${ }^{23,24}$ This discordance may be because of different procedures of mechanical unloading between heterotopic heart transplantation in the animal model and LVAD in a clinical situation.

\section{Limitations}

The current study has some limitations. First, the DCM model in this study was induced by experimental myocarditis, which accompanied LV dilation, systolic dysfunction, and collagen deposition. This animal model may not reflect

TABLE 3. Messenger RNA expressions

\begin{tabular}{lccrc}
\hline & Control & DCM & DCM-UL & P value for DCM vs DCM- \\
& $3.44 \pm 0.38$ & $4.15 \pm 0.27$ & $6.99 \pm 0.75$ & .0051 \\
$\beta_{1}$-AR/GAPDH & $2.50 \pm 0.21$ & $3.40 \pm 0.19$ & $4.76 \pm 0.28$ & .0025 \\
$\beta_{2}$-AR/GAPDH & $1.36 \pm 0.41$ & $9.36 \pm 1.46$ & $14.09 \pm 1.11$ & .0275 \\
Collagen Ia/GAPDH & $0.12 \pm 0.14$ & $7.45 \pm 0.73$ & $9.82 \pm 1.04$ & .0987 \\
Caspase-3/GAPDH & & & \\
\hline
\end{tabular}

$A R$, Adrenergic receptor; $G A P D H$, glyceraldehyde 3-phosphate dehydrogenase; $D C M$, dilated cardiomyopathy; $D C M$ - $U L$, dilated cardiomyopathy (unloaded). 
idiopathic cardiomyopathy exactly; however, it is established and used as a DCM model in other reports. ${ }^{7}$ Therefore, we consider that this model simulates the DCM model. Second, heterotopic transplantation in this study may not replicate the unloading seen in cases in which an LVAD is inserted in a DCM heart. However, we consider this procedure to be a close simulation of LVAD compared with conventional heterotopic transplantation procedures reported previously, given that the LV of transplanted heart is perfused and loaded with a small amount of blood. ${ }^{8}$ However, the possibility remains that the unventilated lungs influence this transplantation model. Third, although heterotopic transplantation involves only a short ischemic time, we consider that the transplantation procedure used in this study can halve ischemic time because of the single anastomosis required compared with that of conventional transplantation. ${ }^{8}$ Moreover, we used cold cardioplegic solution and ice protection during transplantation to minimize the negative effects resulting from ischemia. Fourth, the isolated papillary muscle function test used in this study may not reflect the global function of the LV. However, this procedure is established and represented elsewhere. $^{5,8,9}$ Therefore, we consider this procedure to be one of the functional indicators of the LV. Fifth, in this study, we evaluated mRNA gene expression as a molecular assessment. Therefore, it may not reflect the expression of proteins precisely. However, we believe that the result may help indicate the basic molecular changes. Finally, there is a possibility that the denervation induced by transplantation may have altered the response of $\beta$-ARs. A previous investigation reported that functional and $\beta$-adrenergic density recoveries were independent of cardiac denervation and that other humoral molecules present in the patient with heart failure might influence ventricular remodeling. ${ }^{25}$ Thus, in our opinion there is a possibility that humoral stimulation is one of the factors that contribute to improved $\beta$-AR mRNA expression.

\section{CONCLUSIONS}

Mechanical unloading preserved myocardial contractility and $\beta$-adrenergic response in the DCM rat heart. However, myocardial relaxation was impaired. Furthermore, myocardial atrophy, the ratio of the fibrotic area, and apoptosis all increased with time. These unfavorable responses, although secondary to mechanical unloading, may have a negative impact on a bridge to recovery in DCM patients. Further intervention is needed to preserve cardiac function and prevent myocardial stiffness and apoptosis.

We thank Dr Matsuoka for the physiologic study, Dr Kuwahara, Dr Nakagawa, and Dr Kinoshita (Department of Cardiovascular Medicine) for their molecular techniques, and Ms Kataoka for her histologic techniques.

\section{References}

1. Hetzer R, Muller J, Weng Y, Wallukat G, Spiegelsberger S, Loebe M. Cardiac recovery in dilated cardiomyopathy by unloading with a left ventricular assist device. Ann Thorac Surg. 1999;68:742-9.

2. Dandel M, Weng Y, Siniawski H, Potapov E, Drews T, Lehmkuhl HB, et al. Prediction of cardiac stability after weaning from left ventricular assist devices in patients with idiopathic dilated cardiomyopathy. Circulation. 2008;118(14 Suppl): S94-105.

3. Xydas S, Rosen RS, Ng C, Mercando M, Cohen J, DiTullio M, et al. Mechanical unloading leads to echocardiographic, electrocardiographic, neurohormonal, and histologic recovery. J Heart Lung Transplant. 2006;25:7-15.

4. Tsuneyoshi H, Oriyanhan W, Kanemitsu H, Shiina R, Nishina T, Ikeda T, et al. Heterotopic transplantation of the failing rat heart as a model of left ventricular mechanical unloading toward recovery. ASAIO J. 2005;51:116-120.

5. Oriyanhan W, Tsuneyoshi H, Nishina T, Matsuoka S, Ikeda T, Komeda M. Determination of optimal duration of mechanical unloading for failing hearts to achieve bridge to recovery in a rat heterotopic heart transplantation model. J Heart Lung Transplant. 2007;26:16-23.

6. Wang J, Marui A, Ikeda T, Komeda M. Partial left ventricular unloading reverses contractile dysfunction and helps recover gene expressions in failing rat hearts. Interact Cardiovasc Thorac Surg. 2008;7:27-31.

7. Horii T, Tambara K, Nishimura K, Suma H, Komeda M. Residual fibrosis affects a long-term result of left ventricular volume reduction surgery for dilated cardiomyopathy in a rat experimental study. Eur J Cardiothorac Surg. 2004;26: 1174-1179.

8. Wang J, Tsukashita M, Nishina T, Marui A, Yoshikawa E, Muranaka H, et al. Chronic partial unloading restores beta-adrenergic responsiveness and reverses receptor downregulation in failing rat hearts. J Thorac Cardiovasc Surg. 2009; 137:465-470.

9. Tsuneyoshi H, Oriyanhan W, Kanemitsu H, Shiina R, Nishina T, Matsuoka S, et al. Does the beta2-agonist clenbuterol help to maintain myocardial potential to recover during mechanical unloading? Circulation. 2005;112(9 Suppl): I51-6.

10. Dipla K, Mattiello JA, Jeevanandam V, Houser SR, Margulies KB. Myocyte recovery after mechanical circulatory support in humans with end-stage heart failure. Circulation. 1998;97:2316-22.

11. Zafeiridis A, Jeevanandam V, Houser SR, Margulies KB. Regression of cellular hypertrophy after left ventricular assist device support. Circulation. 1998;98: 656-62.

12. Ogawa D, Tanaka A, Abe K, Olegario P, Kasahara K, Shiraishi Y, et al. Evaluation of cardiac function based on ventricular pressure-volume relationships during assistance with a rotary blood pump. Conf Proc IEEE Eng Med Biol Soc. 2006;1:5378-81

13. Welsh DC, Dipla K, McNulty PH, Mu A, Ojamaa KM, Klein I, et al. Preserved contractile function despite atrophic remodeling in unloaded rat hearts. Am J Physiol Heart Circ Physiol. 2001;281:H1131-6.

14. Klotz S, Barbone A, Reiken S, Holmes JW, Naka Y, Oz MC, et al. Left ventricular assist device support normalizes left and right ventricular beta-adrenergic pathway properties. J Am Coll Cardiol. 2005;45:668-76.

15. Brinks H, Tevaearai H, Muhlfeld C, Bertschi D, Gahl B, Carrel T, et al. Contractile function is preserved in unloaded hearts despite atrophic remodeling. J Thorac Cardiovasc Surg. 2009;137:742-6.

16. Soppa GK, Lee J, Stagg MA, Siedlecka U, Youssef S, Yacoub MH, et al. Prolonged mechanical unloading reduces myofilament sensitivity to calcium and sarcoplasmic reticulum calcium uptake leading to contractile dysfunction. J Heart Lung Transplant. 2008;27:882-9.

17. Bruckner BA, Stetson SJ, Perez-Verdia A, Youker KA, Radovancevic B, Connelly JH, et al. Regression of fibrosis and hypertrophy in failing myocardium following mechanical circulatory support. J Heart Lung Transplant. 2001;20: 457-64.

18. Madigan JD, Barbone A, Choudhri AF, Morales DL, Cai B, Oz MC, et al. Time course of reverse remodeling of the left ventricle during support with a left ventricular assist device. J Thorac Cardiovasc Surg. 2001;121:902-8.

19. Klotz S, Foronjy RF, Dickstein ML, Gu A, Garrelds IM, Danser AH, et al. Mechanical unloading during left ventricular assist device support increases left ventricular collagen cross-linking and myocardial stiffness. Circulation. 2005;112: 364-74.

20. Bruggink AH, van Oosterhout MF, de Jonge N, Ivangh B, van Kuik J, Voorbij RH, et al. Reverse remodeling of the myocardial extracellular matrix after prolonged left ventricular assist device support follows a biphasic pattern. J Heart Lung Transplant. 2006;25:1091-8. 
21. Jugdutt BI, Butler C. Ventricular unloading, tissue angiotensin II, matrix modulation, and function during left ventricular assist device support. J Am Coll Cardiol. 2007;49:1175-7.

22. Schena S, Kurimoto Y, Fukada J, Tack I, Ruiz P, Pang M, et al. Effects of ventricular unloading on apoptosis and atrophy of cardiac myocytes. J Surg Res. 2004; 120:119-26.

23. Bartling B, Milting H, Schumann H, Darmer D, Arusoglu L, Koerner MM, et al. Myocardial gene expression of regulators of myocyte apoptosis and myocyte calcium homeostasis during hemodynamic unloading by ventricular assist devices in patients with end-stage heart failure. Circulation. 1999;100(19 Suppl): II216-23.

24. Milting H, Bartling B, Schumann H, El-Banayosy A, Wlost S, Ruter F, et al. Altered levels of mRNA of apoptosis-mediating genes after mid-term mechanical ventricular support in dilative cardiomyopathy-first results of the Halle Assist Induced Recovery Study (HAIR). Thorac Cardiovasc Surg. 1999;47:48-50.

25. Tevaearai HT, Eckhart AD, Walton GB, Keys JR, Wilson K, Koch WJ. Myocardial gene transfer and overexpression of beta2-adrenergic receptors potentiates the functional recovery of unloaded failing hearts. Circulation. 2002;106:124-9. 